\title{
Optical flow analysis of the ventral cellular layer of the migrating Dictyostelium discoideum slug
}

\author{
Edmond J. Breent and Keith L. Williams \\ Author for correspondence: Keith L. Williams. Tel: +612805 8212. Fax: +6128058174. \\ e-mail: keith.williams@mq.edu.au
}

School of Biological Sciences, Macquarie University, Sydney, NSW 2109, Australia

\begin{abstract}
A digital image analysis system for extracting motion information from timevarying digital light microscopy images is presented. This system is then used to map out the movement profile of the surface layer of cells in contact with the substratum through the extracellular matrix (ECM) of the migrating Dictyostelium discoideum slug. From digital high magnification light microscopy images, the morphology of moving cells within the tail region of a young migrating wild-type WS380B slug is described, and compared with the morphology of streaming $D$. discoideum cells. It is shown that: (i) when the migrating tip of the slug touches the agar substrate, cells in the anterior ventral surface layer of the tip region slow dramatically; (ii) overall cell movement in the ventral surface layer of the migrating $D$. discoideum slug is slower than the movement of the slug as a whole; and (iii) in less than $10 \%$ of cases a wave of movement (groups of cells synchronously slowing down and then accelerating forward) propagates down the slug axis at approx. 1.2 $\mu \mathrm{m}$ $\mathrm{s}^{-1}$. The time interval between waves may be related to the time interval between tip-to-substratum contact that is periodically re-established during normal WS380B slug migration after each aerial projection of the tip.
\end{abstract}

Keywords: Dictyostelium discoideum, motion analysis, cell movement, tissue movement, wave propagation

\section{INTRODUCTION}

At the end of the vegetative phase, the amoebae of Dictyostelium discoideum aggregate together and form into a migrating pseudoplasmodium or slug (Raper, 1940). It has been shown that mature slugs move in a coordinated and periodic fashion : their length remains almost constant (Shaffer, 1964; Inouye \& Takeuchi, 1979; Breen et al., $1992 \mathrm{~b}$ ) and their tip region is lowered and raised at regular intervals from the substratum (Inouye \& Takeuchi, 1979; Vardy et al., 1986; Breen et al., 1987; Vardy, 1992). It is unclear how the coordination and periodic movements of the slug are achieved.

In a bid to understand the internal cellular movements of the slug, Eliott et al. (1991) measured the spatial arrangement of myosin II, a contractile molecule, within

\footnotetext{
† Present address: CSIRO Division of Mathematics and Statistics, Institute of Information Science and Engineering, Locked bag 17, Nth Ryde, NSW 2113 Australia.
}

Abbreviation: ECM, extracellular matrix. cells of migrating slugs and showed that myosin II is peripherally located in 'epithelial' cells, especially at the tip and the rear of the slug. This was consistent with aspects of the 'squeeze-pull' model of slug locomotion (Williams et al., 1986), since it supports the idea that the periphery of the slug contracts and squeezes the slug forward. Siegert \& Weijer (1992), who also considered the internal cellular movements of the slug, have recently argued that the regulation of slug movement is best explained via the principles of aggregation, i.e., wave propagation and chemotaxis (Durston \& Vork, 1979; Clark \& Steck, 1979; Robertson \& Grutsch, 1981; Schaap \& Wang, 1984), rather than the cellular flow model of Odell \& Bonner (1986) or the 'squeeze-pull model' of Williams et al. (1986). In this report we describe how one group of cells within the migrating slug of $D$. discoideum move, and discuss the findings in relation to the above models.

Ultimately, to understand how the slug of $D$. discoideum migrates, requires knowledge of how the cells within the slug move (Williams et al., 1986; Breen \& Williams, 
1988). In particular, this knowledge will be gained, in part, via tracking the internal cellular movements of the slug through its four-dimensional digital representation (Breen \& Williams, 1988). Most conventional approaches for tracking the internal cellular movements of the slug use time-lapse digital light microscopy to record, at known intervals, high magnification sequential images of migrating slug tissue. They also rely on labelling slug cells with vital dyes that predominantly stain prestalk and prestalk-like cells (Sternfeld \& David, 1981). Then a human operator is used to pin-point the positions of stained cells within each image (Durston \& Vork, 1979; Tasaka \& Takeuchi, 1983; Siegert \& Weijer, 1992). Using this method the internal trajectory of each marked cell can be obtained with reasonable accuracy, although it is often difficult to understand how the stained cell in question is moving in three dimensions. Also, this approach is labour intensive and major problems include: only a handful of cells are analysed at any given time; analysis is biased to the movements of prestalk or prestalk-like cells, and the results are not related directly to the movement of the whole slug, i.e. slug tip and tail movements (Breen et al., 1987, 1992a). To automate this process requires digital imaging technology (Breen \& Williams, 1990; Breen et al., 1991), motion analysis procedures (Ullman, 1981; Noble, 1987; Gelles et al., 1988) and a vision system for automatically tracking the movements of unstained tissue within the migrating $D$. discoideum slug. By using such procedures, it has been possible to produce optical flow fields (Horn \& Schunck, 1981) for automatically mapping out the movement of the ventral layer of the migrating $D$. discoideum slug and to discern how the movement of this layer relates to the locomotion of the whole slug.

\section{METHODS}

Slug preparation. $D$. discoideum, strain WS380B, amoebae were grown on SM nutrient agar plates (Sussman, 1966) together with Klebsiella pneumoniae. As the amoebae entered starvation they were harvested in ice-cold water and then washed three times in $1 \mathrm{ml}$ of ice-cold water by centrifuging at 2000 r.p.m. for $2 \mathrm{~min}$. After the last wash and after the supernatant was drained off, $2 \mu \mathrm{l}$ of the amoebae pellet (approx. $3.0 \times 10^{6}$ cells) were pipetted to one side in a Petri dish $(8.5 \mathrm{~cm}$ inner diameter plate) containing water Noble agar $(1.0 \%, \mathrm{w} / \mathrm{v}$; thickness of the agar substrate was approx. $0.5 \mathrm{~mm}$ ) and left to dry for $20 \mathrm{~min}$ in a sterile fume hood. To prevent drying out of the agar substrate, the inner lip of the agar plate was lined with $1 \mathrm{ml}$ of distilled water. Noble agar was used in preference to Calbiochem agar (Breen et al., 1987) as it has superior optical properties. The plates were placed inside black PVC containers with a $3 \mathrm{~mm}$ hole on one side and they were then incubated at $21 \pm 1{ }^{\circ} \mathrm{C}$ in an illuminated room for approx. $16 \mathrm{~h}$. Migrating slugs always formed within $6-8 \mathrm{~h}$.

Video microscopy. A Petri dish (with its lid on) containing migrating slugs was placed on the stage of a Zeiss inverted microscope. The Petri dish was further enclosed in an opaque PVC container that allowed entry of light from above and side light through a $3 \mathrm{~mm}$ hole. Light from above was used to illuminate the slugs for viewing, while light from the side was used to guide slug movement (phototaxis). The light from above was filtered through two Kodak gelatin dark red (640 nm) monochromat filters, cat. no. 1495936. A 40X Zeiss objective was focused onto the ventral side of a slug and images of the ventral surface of the slug were recorded at $3 \mathrm{~s}$ intervals as the slug passed through the field of view. These images were captured with the aid of a Mintron CCD camera and stored on the hard disk (Miniscribe 150Mbytes) of a 386 PC computer (30 MHz) fitted with a Matrox PIP-640 image board. At the same time images of the same scene were stored independently on a Panasonic/National NV8051 time-lapse video recorder set to a compression ratio of $1 / 40$ real-time. The images stored in the computer were used for motion analysis, while the images stored on the video recorder were used for long-term storage and playback. The numerical aperture (NA) of the system was estimated with the aid of a diatom test-slide to be $0.4<\mathrm{NA}<$ $0 \cdot 7$.

Recording motion information from migrating slug tissue. The surface of the slug considered here for motion analysis is the most ventral layer of cells in contact with the substratum through the slime sheath. It is defined by the cells that are in maximum focus nearest to the surface of the agar substrate. From a temporal digital image sequence of this surface, a motion analysis system is used to track the movements of identifiable characteristic optical structures within slug tissue. In broad terms the principle of the tracking procedure is.

(1) The identification of several hundred areas of interest in slug tissue via a filter routine called the top hat box filter (Breen et al., 1991). The top hat box filter is an interest operator for locating compact regions of high image intensity.

(2) After the identification of the areas of interest on the slug surface in the first image these same areas are then located in successive images. This involves searching for areas of greyscale structure in the present image that are similar to those present in the previous image. The search begins in an image area around the original location and several potentially similar areas are identified via an experimentally defined threshold.

(3) The problem is then to identify among the set of corresponding areas of interest the most likely. This is solved by taking into account the behavioural history of the area of interest. The most likely corresponding area is selected based on a weighted combination of similarity in grey-scale, persistence in rate of movement, persistence in direction and for consistency with respect to neighbourhood movements. The weighting is done according to an empirical procedure.

With our system, interest points are generally lost at a rate of approx. $5 \%-6 \%$ per image; therefore appropriate methods for both recovering motion information and for displaying the results for an extended image sequence were required. To do this the velocity field of the image sequence was sampled at predetermined intervals $(15 \mathrm{~s})$ and then the average speed of each sampled velocity field was plotted as a function of time or position within the image sequence. This was done automatically by setting up the top hat box filter algorithm (Breen $e t$ al., 1992b) to locate a unique set of interest points in every fifth frame of the entire sequence. The tracking procedure then mapped each set of interest points into the next frame of the sequence. Via this method sets of velocity vectors (optical flow fields) were collected from each set of interest points located by the box filter algorithm (see below).

From these data, all trajectories for all areas (points) of interest are collected simultaneously in a pair-wise manner via an adaption of the sequential similarity detection algorithm by Barnea \& Silverman (1972) and the disparity analysis of images of Barnard and Thompson (1980). The details of this procedure are described below in order to allow others to use it. Many readers may choose to pass over the next section as it involves a full mathematical description of the approach. 

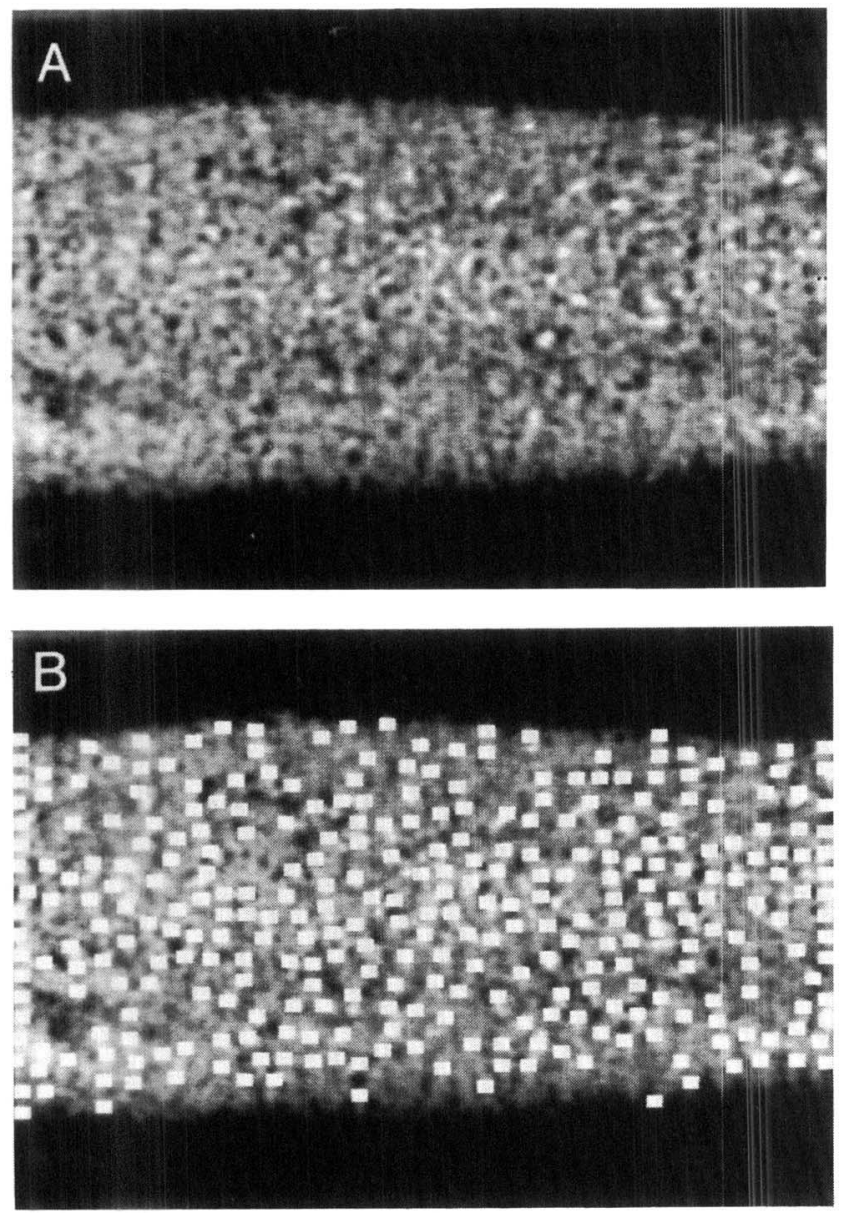

Fig. 1. Locating objects of interest on the ventral cellular surface of a $D$. discoideum slug. (A) Ventral layer of part of a migrating slug of $D$. discoideum. (B) Each white square represents an object of interest located in (A) using the box filter algorithm as described in detail by Breen et al. (1991). Note that objects of interest are not considered to be cells, but simple compact high intensity (grey-scale) regions on the ventral surface. The width of each image is approx. $100 \mu \mathrm{m}$, or approx. 10 cell widths. (Reprinted with permission from J Comput Assisted Microsc 3, 97-101.)

The procedure. First, let a digital video light microscopy image sequence of the ventral surface of a migrating $D$. discoideum slug, collected through a static field of view, be defined as $f(x, y, k)$, where $f(x, y, k)$ represents the light intensity value at location $(x, y)$ within the kth image of the sequence. It will be assumed that the time interval between recorded images within sequence $f$ is held constant, that all images within $f$ have the same twodimensional Euclidean integer coordinate system and are numbered sequentially by a positive integer $k$, starting at zero for the first image of the sequence, i.e. $k \in[0, K-1]$, where $K$ is an arbitrary large positive integer and represents the number of images in the entire sequence.

To track the movements of the slug tissue, points of interest (identifiable characteristic optical structures) are initially located within an image via the top hat box filter (Breen $e t$ al., 1991) as seen in Fig. 1. Let a set of interest points within any image be defined as $P^{k}=\left\{\boldsymbol{x}^{i k}\right\}$, where $\boldsymbol{x}^{i k}$ denotes the location or centroid, $\left(\boldsymbol{x}^{i k} \boldsymbol{y}^{i k}\right)$, of the $i$ th point of interest in the kth image. Since it is assumed that $k$ varies constantly with time, $\boldsymbol{u}^{i k}=\boldsymbol{x}^{i k+1}-\boldsymbol{x}^{i k}$ can be used to gain an estimate of the instantaneous velocity of the $i$ th interest point at time $k$. Hence, by solving sequentially for $\boldsymbol{u}$, a trajectory, $T^{i k}=\left[\boldsymbol{u}^{i k}, \boldsymbol{u}^{i k+1}, \ldots, \boldsymbol{u}^{i k+K^{i}-1}\right]$, can be built up starting at each point $\boldsymbol{x}^{i k} \in \boldsymbol{P}^{k}$, where $K^{i}$ is used to represent the number of images that the $i$ th interest point is located in.

To map a single interest point from one image into the next, a small rectangular image region (here, $13^{2}$ picture elements) centred at the interest point, $\boldsymbol{x}^{i k}$, is used to form a reference image $\boldsymbol{r}^{i k}$. Let $r^{i}\left(x_{2}, k_{\text {. }}\right)$ denote the image intensity at location $(x, y) \in r^{i k}$. Next, centred at the same location in the next image, $k+1$, a rectangular search plane, $s^{i k+1}$, is defined and likewise, $s^{i}(x, y, k+1)$ is used to denote its image intensities. The search plane, $s$, is always larger (here, $21^{2}$ picture elements) than $r$ and its size is a function of the temporal resolution of the image sequence. The object now is to locate the position of each reference image within each search image. Therefore, let $(u, v)$ be any offset vector from the centre of $s^{i k+1}$ that will position the search plane with respect to the reference image such that $r^{i k} \subset$ $s^{i k+1}$. Since the size of the reference image and the search plane is held constant for all interest points, $D$ is used to represent the set of all possible offset vectors $\{(u, v)\}$, i.e. $D=\{(u, v) \mid$ $\left.\left(x+u_{2} y+v\right) \in s, \forall(x, y) \in r\right\}$. Now, for all $(u, v) \in D$, the absolute grey-scale differences between the picture elements of the reference image, $r^{i k}$, and those of the search plane, $s^{i k+1}$, are determined via

$d^{i k}(u, v)=\sum_{(x, y) \in r^{i k}}\left|r^{i}\left(x_{2} y, k\right)-s^{i}\left(x+u_{2} y+v, k+1\right)\right|$

In practice, the differences are not accumulated for all picture elements $(x, y)$ of the reference image, $r$. Instead the $j$ first picture elements are scanned in random order. As a cumulative difference, as defined by $d^{i k j}(u, v)$, is computed, it is tested against the threshold value $\operatorname{Th}(j), j \in[0, \# r-1]$, where $\# r=13^{2}$. If $d^{i k j}(u, v)>\operatorname{Th}(j)$ the comparison stops and the vector $(u, v)$ is rejected and the next member of $D$ is tested for similarity. The threshold function used here is defined by $\operatorname{Th}(j)=\delta j+\delta$, where $\delta$ is a positive real value and represents the level of deviation or noise per picture element. $\delta$ is determined empirically and here $\delta=4 \cdot 0$. In this way all members of $D$ are tested against all members of $P^{k}$. Let $U^{i k}=\left\{(u, v) \mid d^{i k \# r-1}(u, v) \leqslant\right.$ Th $(\# r-1)\}$ be the set of candidate disparity vectors collected, as just described, for the $i$ th interest point and let $\# U^{i k}$ be the number of vectors in this set. The problem now is to pick the most likely vector $(u, v) \in U^{i k}$ to form $\boldsymbol{u}^{i k}$. To do this, for all $(u, v)$ $\in U^{i k}$ where \# $U^{i k} \neq 0$ a probability value, $p$, of being correct is determined. The vector $(u, v)$ with the highest probability of being correct is then chosen from each $U^{i k}$. This is accomplished via the following matching approach.

Let $M(i, k, u, v)$ represent a matching value between interest point $\boldsymbol{x}^{i k} \in P^{k}$ and vector $(u, v) \in U^{i k}$ as

$M(i, k, u, v)=\sum w c(i, k, u, v)$

where each $w$ is a weight and each $c$ is a matching criterion value, which is also a function of $(i, k, u, v)$. The matching criteria values, $c$, used here are defined as

$c_{1}(i, k, u, v)=\frac{\# r}{\# r+d^{i k \# r-1}(u, v)}$
$c_{2}(i, k, u, v)=\frac{2 \sqrt{\left\|\boldsymbol{u}^{i k-1}\right\|\|(u, v)\|}}{\left\|\boldsymbol{u}^{i k-1}\right\|+\|(u, v)\|}$
$c_{3}(i, k, u, v)=\frac{1}{2}\left(1+\frac{\boldsymbol{u}^{i k-1} \cdot(u, v)}{\left\|\boldsymbol{u}^{i k-1}\right\|\|(u, v)\|}\right)$ 
where $\|(u, v)\|=\sqrt{u^{2}+v^{2}}$. Now, $c_{1}$ is used to measure the spatial optical similarity between two areas and is 1 if the two areas are $100 \%$ identical, while $c_{2}$ and $c_{3}$ measure the speed and the directional coherence respectively of the interest point being tracked. They represent inertia (see also Sethi \& Jain, 1987); hence both $c_{2}$ and $c_{3}$ will equal 1 if there is no change in an interest point's motion characteristics. Note that during the mapping of interest points from image $k=0$ to $k=1, c_{2}$ and $c_{3}$ cannot be evaluated. This occurs for the first two images within the sequence or if interest points are tracked across only two arbitrary images $M(i, k, u, v)=c_{1}(i, k, u, v)$. Otherwise $M(i, k, u, v)$ is determined from eq. (2) using the following three weights: $w_{1}=0.8, w_{2}=0.18$ and $w_{3}=0 \cdot 02$. These particular values of $w$ were obtained empirically, via (1) getting the system to track correctly optical structures within migrating $D$. discoideum slug tissue that were identified by eye, and (2) by checking the resultant movement profiles visually with the same recorded sequence of a migrating $D$. discoideum slug collected on video tape.

Note that when $c \in[0,1]$ and $\Sigma w=1$, as is the case here, it ensures that $M \in[0,1]$, but it does not ensure that $\Sigma M=1$, nor does it define the null case, i.e. when there is no correct match. Therefore, the values of $M$ cannot be used as probability values, $p$. An initial probability of correctness can be tentatively assigned to each $(u, v) \in U^{i k}$ using Bayes' rule in the form

$p^{0}(i, k, u, v)=\left(\frac{M(i, k, u, v)}{\sum_{(u, v) \in U^{i k}} M(i, k, u, v)}\right) \max \{M(i, k, u, v)\}$

This equation assumes that in most cases the vector with maximum $M$ is the correct one and that the probability that no vector in set $U^{i k}$ can be correctly assigned to point $\boldsymbol{x}^{i k}$ is given by $p^{0}(i, k)=1-\max \left\{M(i, k, u, v) \mid(u, v) \in U^{i k}\right\}$. While useful, these initial probabilities do not consider how the interest points are moving as a cohort. Therefore, it is proposed that they car be improved by introducing the movements of neighbouring interest points into the procedure. To do this, let $\operatorname{Nbr}\left(\boldsymbol{x}^{i k}\right)=$ $\left\{\boldsymbol{x}^{j k} \mid \boldsymbol{x}^{j k} \in P^{k}, \max \left\{\left|x^{i k}-x^{j k}\right|,\left|y^{i k}-y^{j k}\right|\right\}<20, j \neq i\right\}$ represent the neighbourhood set of interest points surrounding point $\boldsymbol{x}^{i k}$ and let $C\left(\boldsymbol{x}^{i k}, u, v\right)=\left\{(j, m, n) \mid \boldsymbol{x}^{j k} \in \operatorname{Nbr}\left(\boldsymbol{x}^{i k}\right),(m, n) \in U^{j k}\right.$, max $\{|u-m|,|v-n|\}<2\}$ be the set that addresses the offset vectors within the neighbourhood of $\boldsymbol{x}^{i k}$ that are considered to be consistent with vector $(u, v) \in U^{i k}$. For this study, a distance of 20 picture elements represents approx. $10 \mu \mathrm{m}$ or one cell diameter. The initial probabilities are iteratively modified by the following updating rule

$p^{t+1}(i, k, u, v)=\beta\left(p^{t}(i, k, u, \nu)\left[1+\alpha q^{t}(i, k, u, v)\right]\right)$

where

$q^{t}(i, k, u, v)=\sum_{(j, m, n) \in C\left(x^{i k}, u, v\right)} p^{t}(j, k, m, n)$

$\alpha$ is an adjustable gain (here, $\alpha=3$ ) and $\beta$ is a normalizing function, such as eq. (4), that assures that the sum of the probabilities equals one after each iteration, i.e.

$p^{t}(i, k)+\sum_{(u, v) \in U^{i k}} p^{t}(i, k, u, v)=1$

Note also that the above updating formula, eq. (5), does not alter a vector's probability if $q^{t}(i, k, u, v)=0$ for all $(u, v) \in U^{i k}$. This is a useful feature for when the system is tracking a point of interest that is moving against the general flow of its neighbours. After four iterations of the above updating step the disparity vector, $(u, v)$, with the highest probability from each set $U^{i k}$ is selected. Let these vectors with the highest probability be designated individually as $\boldsymbol{b}^{i k}$, i.e. $\boldsymbol{b}^{i k} \in U^{i k}$.
The above procedure only provides the position of best fit as a discrete value, i.e. grid position, and for long range tracking this creates a rounding-off problem (error). Therefore, the system automatically adjusts for subpixel alignment between the reference image and its corresponding position in the search plane of the next image. To do this, a $3 \times 3$ difference matrix is formed by recording the sum of the absolute differences between the reference image centred at each position within a $3^{2}$ neighbourhood of its corresponding grid position in the next image of the sequence. A two-dimensional quadratic function is then fitted to these values and differentiated to locate the minimum. The position of the minimum, if contained within this neighbourhood, is then assigned as the true matching location of the reference image within the search plane, or else the corresponding location is not altered.

After subpixel alignment has been used to improve the estimated offset vector $\boldsymbol{b}^{i k}$, it is used to update each trajectory $T^{i}$ and to obtain the next set of interest points $P^{k+1}=\left\{x^{i k+1}\right\}$, i.e.

$\boldsymbol{x}^{i k+1}=\boldsymbol{x}^{i k}+\boldsymbol{b}^{i k}$

$\boldsymbol{u}^{i k}=\boldsymbol{b}^{i k}$

The set of corresponding interest points, $P^{k+1}$, are then mapped into image $k+2$ and so on. This process continues until the end of the image sequence is reached or until all interest points initially assigned for tracking, via the top hat box filter routine (Breen et al., 1991), have been lost, i.e. fail to get matched to a corresponding position within another image.

Salient features of the motion analysis system. The motion analysis system just described is fast, since it uses dynamic arrays (Breen et al., 1992b). It takes approximately $1 \mathrm{~min}$, on a $30 \mathrm{MHz}$ 386 PC fitted with a Matrox PIP-640 image board, to map 200-300 interest points across two images. The following points highlight the salient features of the motion analysis system developed.

(1) Objects or interest points are allowed to be lost, such as when they move out of the field of view, or plane of focus.

(2) The shape, texture and contrast of an interest point can change during tracking, but only slowly and not to any extent between two sequential images; otherwise the system will fail to locate it (i.e. lose it).

(3) The system solves the correspondence problem (Ullman, 1981) via relaxation labelling, i.e. it uses neighbourhood analysis to eliminate ambiguity (Rosenfeld et al., 1976).

(4) It does not require special processing of a cellular surface, such as staining or the addition of markers to be able to analyse the movement of the surface.

(5) It is fully automated, and therefore helps to provide an unbiased estimate of the overall pattern of movement within a time-varying scene.

\section{RESULTS}

\section{Filming the movement of the entire ventral surface layer of the migrating slug}

Because slug movement was analysed using high magnification digital light microscopy, it meant that the view of the slug was restricted to only a $120 \times 120 \mu \mathrm{m}^{2}$ square of the slug surface at any given instant in time. To help overcome this limitation, a filming chamber was constructed to record images of the ventral layer of a moving $D$. discoideum slug at $3 \mathrm{~s}$ intervals, while it migrated through a static field of view (see Fig. 2). Under the filming conditions, the entire slug generally moved 


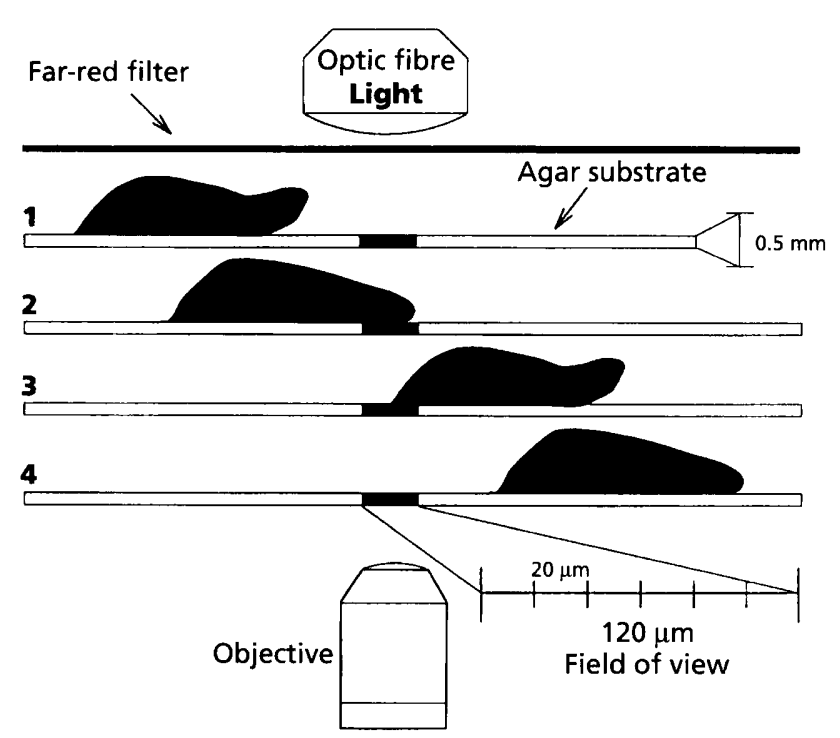

Fig. 2. Data collection strategy. In this schematic representation we have depicted the same slug from the side at four different stages. Stage (1) represents the slug just prior to its tip landing on the agar viewing window (hatched area). Stage (2) represents the slug just after its tip has landed on the agar substrate (dark region). Stage (3) represents the slug just after it has migrated through our field of view. Stage (4) represents the slug continuing to migrate beyond the field of view. When the tip lands on the agar and it is within the field of view, we start collecting images of the slug's ventral surface layer at $3 \mathrm{~s}$ intervals (stage 2). Image collection continues until the entire slug has moved through the field of view or until approx. 60 min of image collection (stage 3). Note also that we have depicted the field of view at stage (4) to be divided into six non-overlapping slices (sub-fields of view).

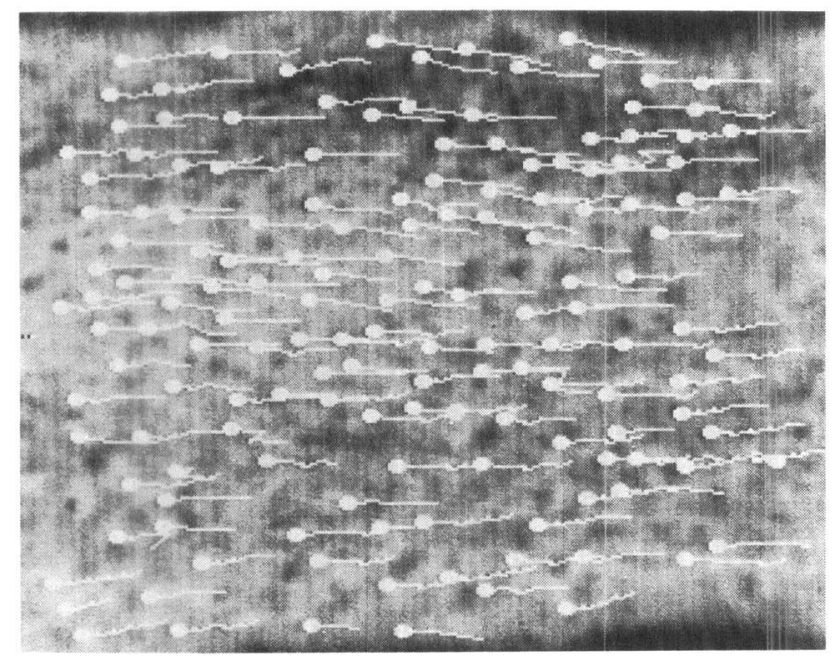

Fig. 3. Tracking analysis example. A portion of the ventral surface of a migrating $D$. discoideum slug moving from left to right. Each line drawn represents the trajectory for $15 \mathrm{~s}$ of movement of an interest point (solid circles). Width of image is approx. $120 \mu \mathrm{m}$ or approx. 12 cell lengths.

through the observation window. To ensure that slug behaviour was relatively normal during image capture, the tip of the slug was located after recording, to see in
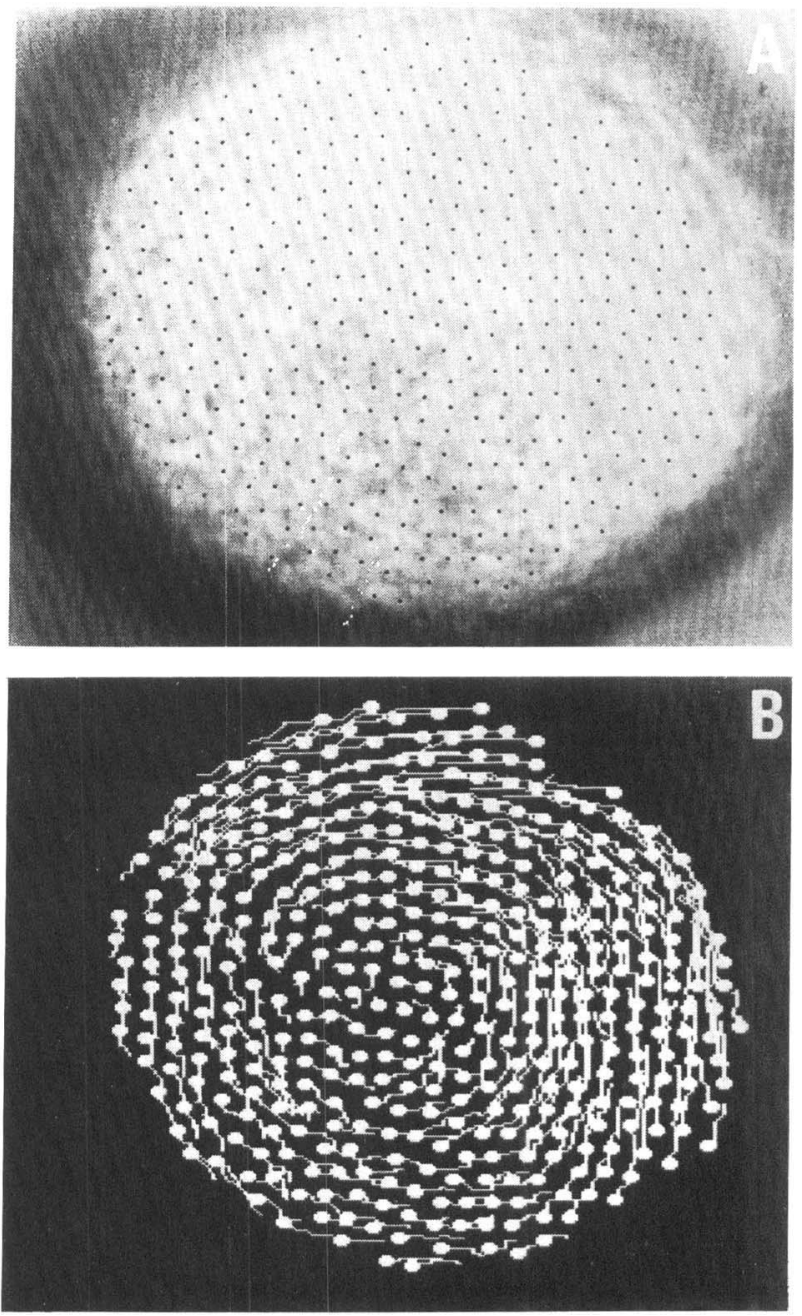

Fig. 4. Tracking the movements of the dorsal surface from a developing WS380B mound. (A) Overhead view of the tip region from a developing WS380B mound. Each black dot represents the centre of a $13 \times 13$ pixel region of interest located by the top hat box filter (see Fig. 1). (B) Trajectories for points located in (A) after $24 \mathrm{~s}$ of movement. Width of image is approx. $120 \mu \mathrm{m}$.

what direction and how far it had moved. This second round of observations confirmed that the slug continued migrating normally (i.e. it did not enter culmination just beyond the field of view) and that it did not undergo any significant turn during image recording. If the slug turned during analysis this would have complicated the study of movement of the internal cells, especially if they moved in a planar or spiral fashion. Also, it was important that the slug did not enter culmination, as it has already been shown that periodic movements are observed during culmination (Durston et al., 1976; see also Deml Rand \& Sussman, 1983). If these conditions were met, the sequence was then marked as a reliable migration sequence and analysed for motion information.

Further, it was important to (i) synchronize the analysis between different slugs examined, as this would allow comparisons to be more easily made between different 

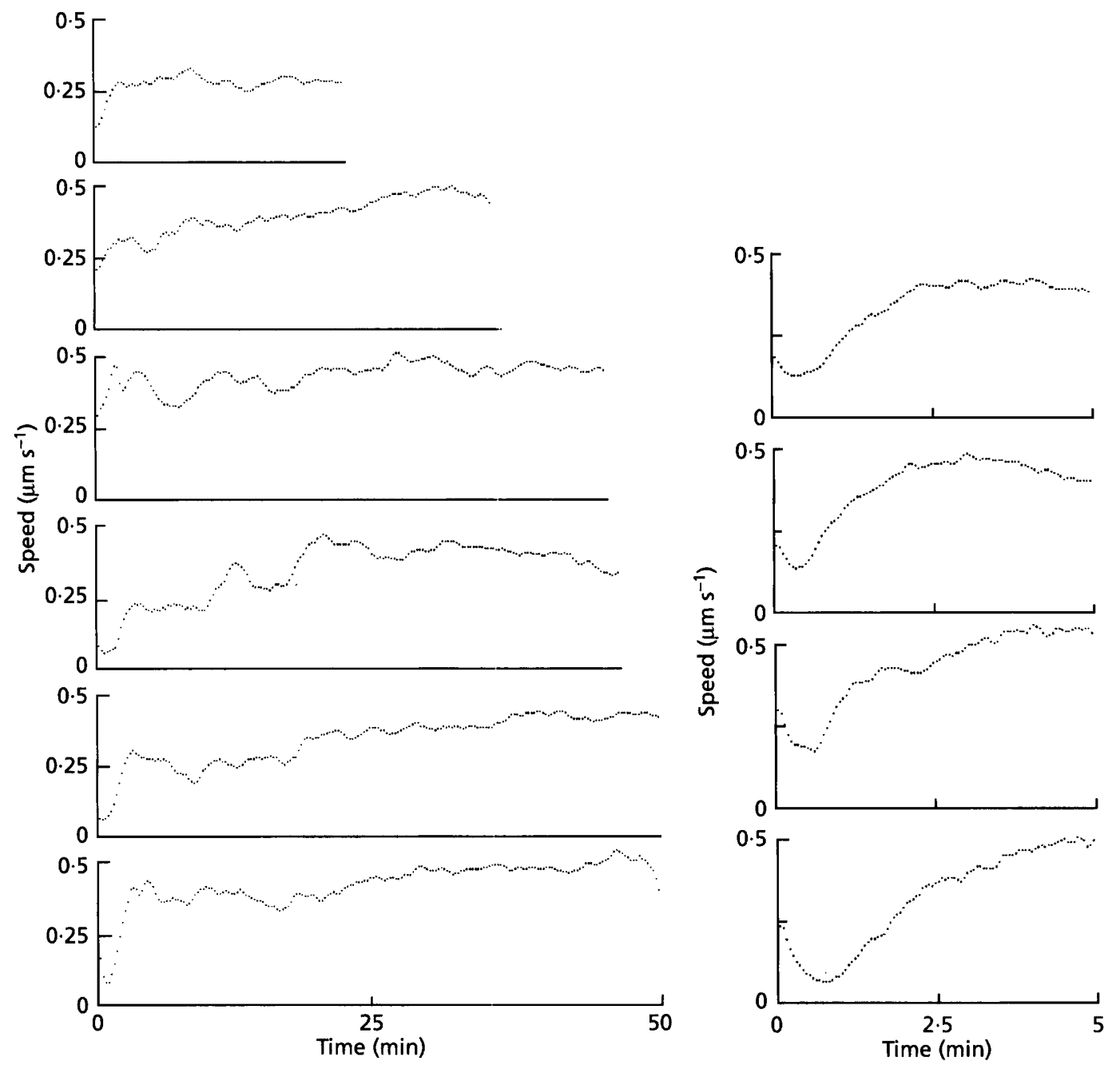

Fig. 5. Movement profiles of the entire ventral layer of six different slugs. Time zero coincides with the tip of each slug landing on the agar substrate. Each point represents the average speed of the ventral layer passing over the field of view at $15 \mathrm{~s}$ intervals. The sequence ends when the rear of the slug passes over the viewing window.

Fig. 6. Movement profiles of the anterior ventral layer of four different slugs. Time zero is the time of tip landing on the viewing surface.

slugs and (ii) see if the cells in the tip region when in contact with the substratum (through the sheath) came to a stop as predicted by the 'squeeze-pull' model of slug locomotion (Williams et al., 1986). To do this, images of the ventral anterior surface of the migrating slug were collected from the time it came into contact with the substratum. It was a relatively simple task to discern visually when the tip touched down on the agar using an inverted light microscope. The complete scheme used for collecting a time-varying image of the migrating $D$. discoideum slug's entire ventral cellular surface is outlined in Fig. 2.

Fig. 3 represents a segment of the ventral surface of a moving $D$. discoideum slug. The white lines overlaid on this image represent the trajectories of the interest points whose initial position is highlighted as a small solid circle at the beginning of each line. Note, movement is from the left to right and each trajectory represents $15 \mathrm{~s}$ (five measurements) of movement recovered from an image sequence, where each image was recorded at $3 \mathrm{~s}$ intervals. These trajectories demonstrate that the motion of the component parts of the ventral surface is essentially parallel with respect to the edge of the slug. This is a consistent phenomenon that has been observed regardless of the distance of the segment from the tip of the slug. Note, however, that only movement of cells attached to the agar was filmed and so this does not include movement of cells in the aerial section of the tip. Thus, movement of the ventral surface layer of the slug that is in touch with the agar appears to be planar. The average speed recorded for the surface, in Fig. 3, was $0.67 \mu \mathrm{m} \mathrm{s}^{-1}$, standard deviation $0.08 \mu \mathrm{m} \mathrm{s}^{-1}$, average displacement along the $x$-axis $10 \cdot 0 \mu \mathrm{m}$, along the $y$-axis $1.3 \mu \mathrm{m}$. Also, $75 \%$ of the 
Table 1. Comparison of the speed of the tip versus speed of the entire ventral layer for 10 slugs

The average speed of the entire ventral layer was compiled by taking the average of all the velocity vectors recorded at $15 \mathrm{~s}$ intervals as the entire slug moved through the viewing window. Typically, 4000-8000 individual velocity vectors were recorded for each slug and considered in estimating this value.

\begin{tabular}{|c|c|c|}
\hline $\begin{array}{l}\text { Tip speed } \\
\left(\mu \mathrm{m} \mathrm{s}^{-1}\right)\end{array}$ & $\begin{array}{c}\text { Average speed } \\
\text { of ventral layer } \\
\left.\qquad(\mu \mathrm{m} \mathrm{s})^{-1}\right)\end{array}$ & $\frac{\text { Ventral speed }}{\text { Tip speed }}$ \\
\hline $0 \cdot 31$ & $0 \cdot 28$ & 0.90 \\
\hline $0 \cdot 50$ & $0 \cdot 40$ & 0.80 \\
\hline $0 \cdot 40$ & 0.34 & 0.85 \\
\hline 0.57 & 0.42 & 0.74 \\
\hline $0 \cdot 25$ & $0 \cdot 18$ & 0.72 \\
\hline $0 \cdot 27$ & $0 \cdot 26$ & 0.96 \\
\hline $0 \cdot 35$ & 0.29 & 0.83 \\
\hline $0 \cdot 30$ & 0.25 & $0 \cdot 83$ \\
\hline $0 \cdot 36$ & $0 \cdot 30$ & 0.83 \\
\hline 0.40 & $0 \cdot 44$ & $1 \cdot 10$ \\
\hline
\end{tabular}

initial 212 points set for tracking were successfully mapped across all five images, i.e. some $25 \%$ of points were lost during the analysis.
Others have shown that movement at the tip of $D$. discoideum slug involves spiral motion (Siegert \& Weijer, 1992). We demonstrated that our method detects spiral motion of cells by studying aggregating mounds. Fig. 4A is an overhead view that shows the tip region of a developing WS380B mound. Each black dot overlaid on Fig. 4A represents the centre point of a region of interest that was automatically located via the top hat box filter (Fig. 1). Fig. 4B displays the trajectory of these points for $24 \mathrm{~s}$ of movement and the average velocity of the outer ring of the spiral is $0.5 \mu \mathrm{m} \mathrm{s}^{-1}$. As can be seen from Fig. 4B the velocity of the spiral reduces towards the centre of the mound.

\section{Movement of the ventral layer of the migrating slug}

Fig. 5 displays the average speed of flow of the ventral cellular slug surface through the field of view for six slugs. Time zero coincides approximately with the tip of the migrating slug landing on the substratum. Note: (i) that each plot reveals the tip of the slug slowing after landing on the substratum, (ii) after a short period, in which the ventral layer in the anterior region slows, it then accelerates rapidly and (iii) the plots do not display convincing evidence of periodic oscillations.

To investigate more closely the event of tip landing on the substratum and to test whether the tip of a migrating slug actually stopped when it came into contact with the
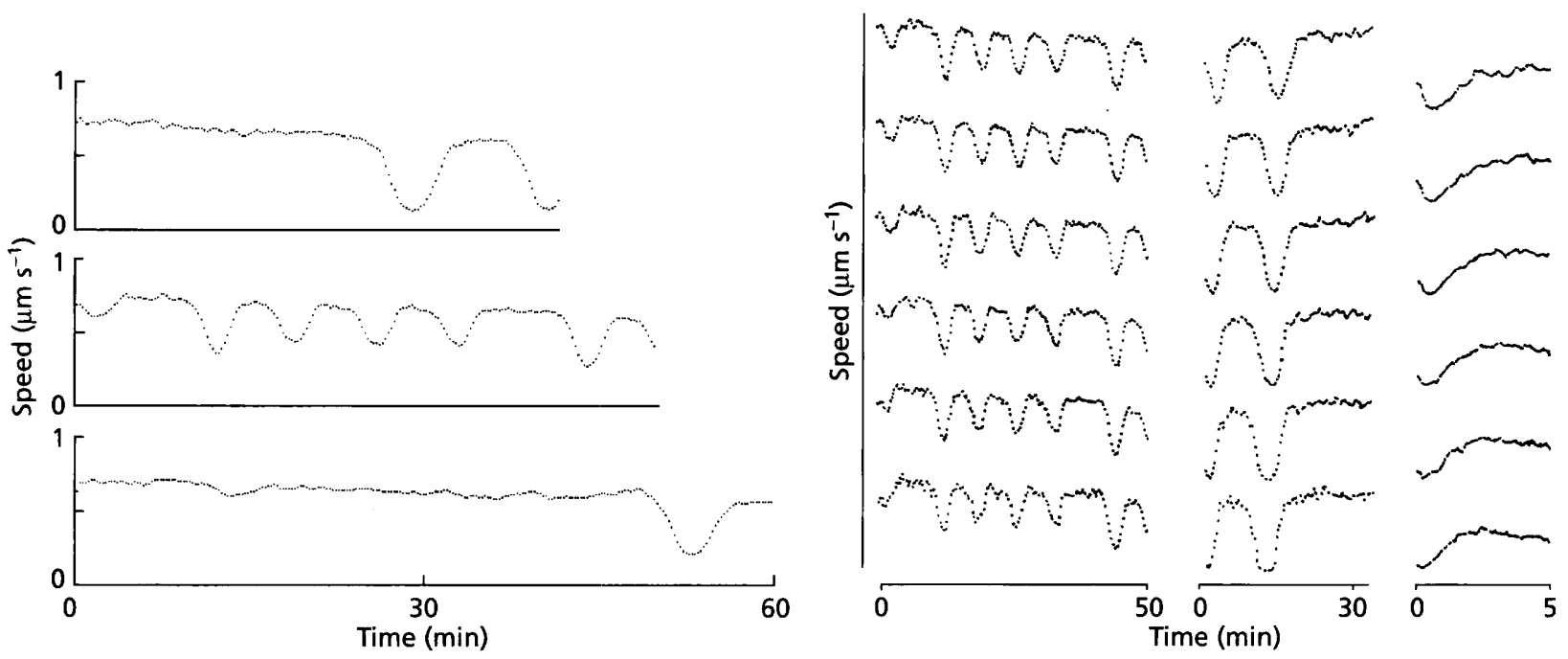

Fig. 7. Pulses of movement along the long axis of the slug. Time zero coincides with the start of data collection which unlike the previous results shown in Fig. 5 and Fig. 6 does not coincide with the tip of the slug touching down on the agar substratum.

Fig. 8. Propagation of waves of movement along the slug axis. Here, the field of view (Fig. 2, stage 4) has been divided into six non-overlapping slices of approx. $20 \mu \mathrm{m}$ in width. Each column represents six movement profiles and three slugs are displayed in this figure. Note that the first column is from the middle profile shown in Fig. 7 , the second column is from the first profile shown in Fig. 7 and the third column is from the first profile given in Fig. 6 . Slug movement is from left to right. The bottom movement profile in each of the three columns represents the speed of movement detected at the far right-hand side of the field of view, while the top profile represents the speed of movement detected at the far left-hand side of the field of view. Note that (i) by comparing the bottom profile with those profiles directly above it, it can be seen that these wave forms propagate along the long axis of the slug and (ii) only about $10 \%$ of the data analysed during the course of this work displayed such clear pulses; $90 \%$ of the profiles collected and analysed are best represented by those given in Fig. 5 . 
substratum, a further four slugs are considered. In this experiment the sampling rate was set at 3 s intervals rather than the coarser interval of $15 \mathrm{~s}$ (Fig. 5). Fig. 6 displays these results and it clearly shows that as the tip region comes into contact with the substratum the ventral cells slow down dramatically and then rapidly accelerate. Note that in the most anterior portion of the touch down zone, cells appear to slow down essentially on contact, while at the rear of the touch down zone (e.g. the cells at least $100 \mu \mathrm{m}$ back from the tip) cells reach a minimum speed after approx. $1 \mathrm{~min}$.

A second piece of information that can be gleaned from this type of analysis is the difference between the average speed of the entire ventral layer of the slug as it moves through the observation window to that of the slug as a whole. The speed of the whole slug can be measured by determining the position of the tip of the slug just prior to image recording and then determining its position after image recording (50-60 min later). The displacement distance of the tip over the time interval provides an estimate of the net translational speed of the tip of the slug. Table 1 shows that our analysis reveals, except in one case, that the ventral layer of the slug moves forward slower than the tip of the slug.

\section{Pulses of cellular movements along the slug axis}

To further investigate the claim that cells in slugs move periodically as seen during aggregation (Siegert \& Weijer, 1992) we examined the movements of the ventral surface layer of slugs that were typically longer than average (i.e. young WS380B slugs were analysed, Breen et al., 1992a) and by starting image recording at approximately half way along the slug axis rather than at the very tip of the slug.

Fig. 7 shows the results of starting image recording at half way along the length of the slug rather than at its tip region. As seen in Fig. 7 pulses of movement are convincingly displayed. The top and bottom plots displayed in Fig. 7 show that pulses of movement were not detected until approximately 30 and 50 min after image recording had begun. Although there is only one clear pulse displayed in the bottom plot of Fig. 7, we know that a further three pulses occurred at approximately $10 \mathrm{~min}$ intervals after the 60 min mark, since a video recording was collected of the same slug, which extended our viewing time, but not our analysis, beyond what could be captured and stored on the computer's hard disk. Fig. 7 shows that for one slug, when pulses of movement are observed they appear at $8-10 \mathrm{~min}$ intervals or at a frequency of approx. $0 \cdot 1 \mathrm{~min}^{-1}$, which is also comparable to the frequency of tip raising and lowering previously documented for a shuffling mature WS380B slug (Breen et al., 1987).

Because the field of view was digitized (Fig. 2), it was easy to analyse the flow of movement of the ventral layer at several positions within this field and independently of other positions. Then by correlating the output of the motion analysis system from each of these positions, it was hoped that it would be possible to both detect a travelling wave of movement and measure its speed of

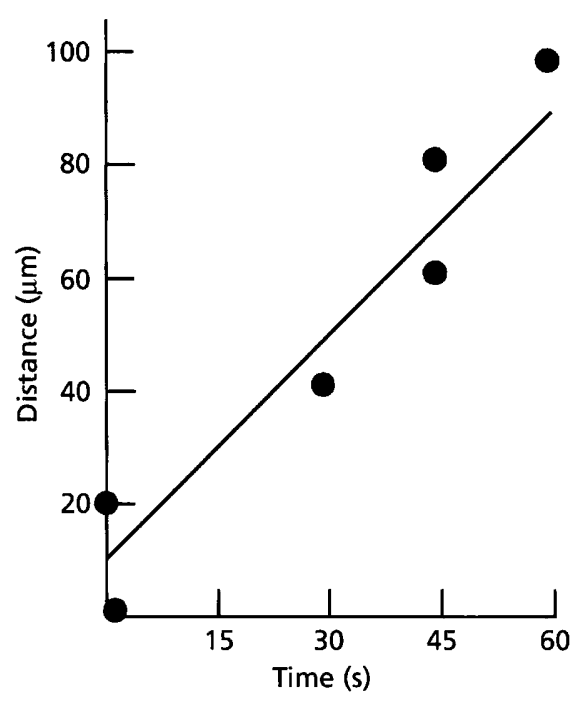

Fig. 9. Cross-correlation. Here we plot the results of correlating the bottom signal in Fig. 8 (first column) with itself (autocorrelation) and the five signals directly above it (crosscorrelation). The time shifts representing the maximum correlation for each signal have been plotted in seconds on the $x$-axis. The centre-to-centre distance between each signal and the first signal has been plotted on the $y$-axis. To these points we fit a least squares regression line and from the slope of this line we obtain the speed of propagation of the signal along the slug axis. Note for this signal we obtain $1.33 \mu \mathrm{m} \mathrm{s}^{-1}$.

propagation. This was accomplished by dividing the field of view (each image) into six equally spaced columns of the same width (thus producing six observation windows of size $20 \times 120 \mu^{2}$ ) and by measuring the average speed of cellular flow through each of these windows independently.

Fig. 8 displays a more complete set of results collected from three of the slugs that appear in Figs 6 and 7. Each column in Fig. 8 represents the six outputs from each sequence; the bottom plot in each column represents the average speed of cellular flow through the far right-hand column of the field of view, while the top plot in each column represents the average speed of cellular flow through the far left-hand column of the field of view. The first two columns show propagation of multiple waves backwards along the slug. These sequences occurred in the middle of slugs. The third column displayed in Fig. 8 shows that the slowing down of the most ventral cells when the tip first touches the substratum also propagates back along the slug's axis, although only a single wave is observed.

By cross-correlating the bottom plot in each column displayed in Fig. 8 with the plots above, it is possible to determine accurately the speed of propagation of these wave forms. The speed of wave propagation was determined as shown for one example in Fig. 9. From the results given in Fig. 8 the speed of wave propagation along the slug axis is estimated to be on average $1.2 \mu \mathrm{m}$ $\mathrm{s}^{-1}$, which in turn is typically $2-3$ times faster than the average speed of a migrating slug (Table 1). 

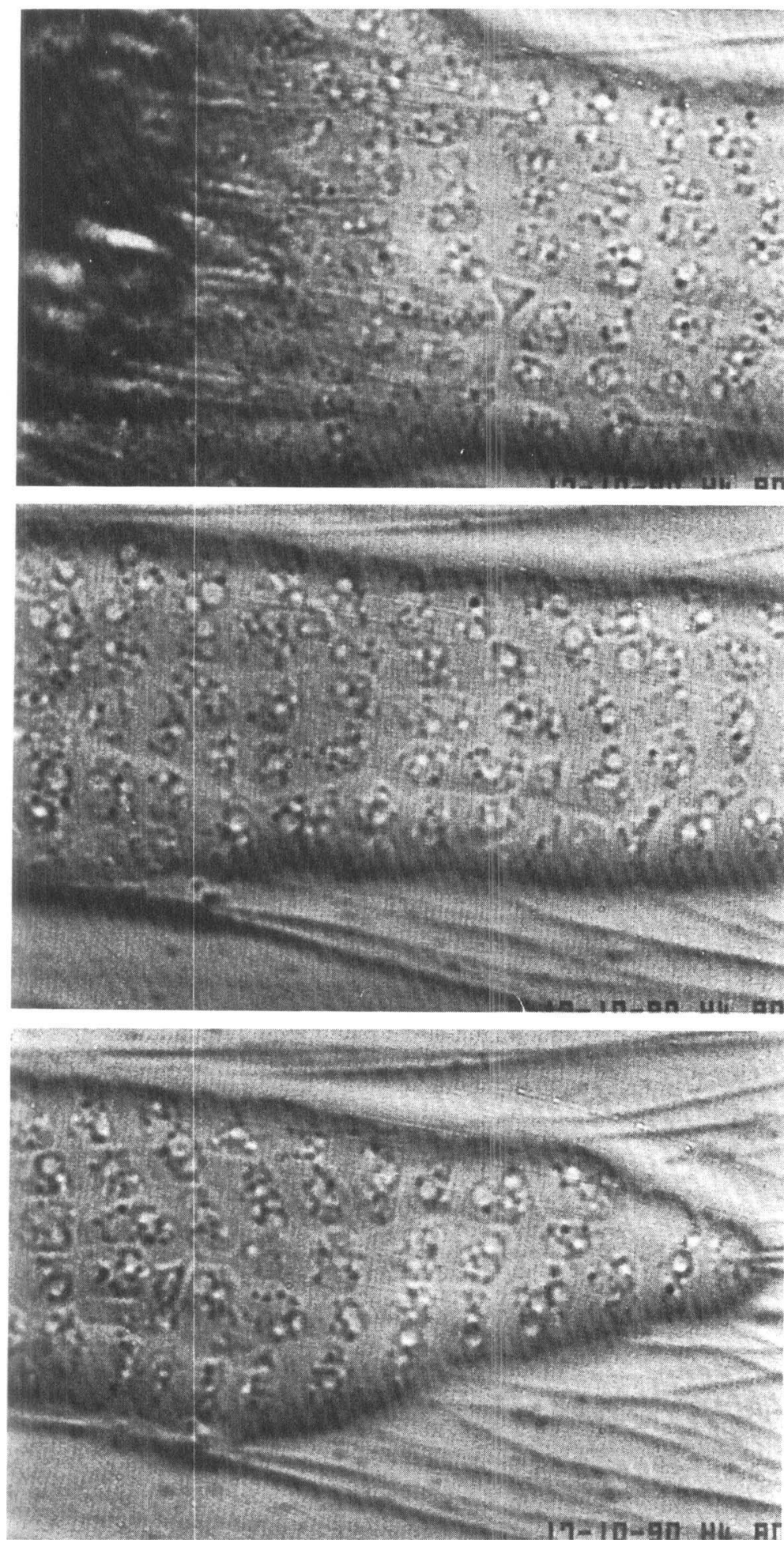

Fig. 10. Tail region of a young migrating WS380B slug. The width of each image is approximately $115 \mu \mathrm{m}$. In the left part of the top image the rear of the body of the slug is just visible. Each image continues from the one above it, and therefore the length of this tail is approx. $350 \mu \mathrm{m}$.

\section{The tail region of a young WS380B slug}

Young WS380B slugs transform into the mature form by compacting their rears. The tail of a young WS380B slug is quite long $(1-2 \mathrm{~mm})$ and it can often be just one cell layer in thickness as shown in Fig. 10. From a visual analysis of time-lapse video recordings of the movement of the cells within the tail region of young migrating slugs, it is apparent that there is a supracellular organization between cells. This organization is easily distinguished as transverse bands across the tail region (Fig. 10). The banding pattern results from the lining up of the cytoplasmic particles in each cell across the width of the tail. We have also discovered that these cells show polarity in the sense that their anterior half resembles a single large pseudopod which is granule free (hyaline ectoplasm), 
while their posterior half contains the cytoplasmic particles and the more granular endoplasm. Although ectoplasm appears to entirely surround the endoplasm of each cell, it is more readily visualized in the leading half of each cell within the tail region. The morphology of the cells revealed in Fig. 10 is more rounded than that displayed by streaming aggregating $D$. discoideum cells, which are typically 5 times longer than their widths (Bonner, 1947). This morphological change has already been described in molecular terms by Siu et al. (1988) and is believed to reflect the loss of contact sites-A (gp80), a cell surface glycoprotein, from the cellular membrane of each aggregating cell as they enter the aggregation mound and by the loss of filopodia.

\section{DISCUSSION}

In this report a new digital imaging vision system is presented for measuring the motion characteristics of unmarked cellular surfaces; it is applied to studying the movement of the ventral surface layer of the migrating $D$. discoideum slug. Firstly, it was shown that when the advancing tip of the slug touches the agar its ventral surface layer slows dramatically. Next it was shown that the ventral surface layer of the slug generally moves forward at a rate $10-20 \%$ slower than that of the whole slug, and thirdly, it was demonstrated that waves of movement can be propagated along the slug axis and at a rate of approx. $1 \cdot 2 \mu \mathrm{m} \mathrm{s}^{-1}$.

Earlier studies (Durston \& Vork, 1979; Siegert \& Weijer, 1991, 1992) showed that a few cells within the slug move in a pulsating manner. Durston \& Vork (1979) estimated that waves of movement propagate along the slug axis at a rate of $0.8 \mu \mathrm{m} \mathrm{s}^{-1}$, which is comparable to the estimate provided here of $1.2 \mu \mathrm{m} \mathrm{s}^{-1}$. These studies have also shown that individual cells move at a relatively slow speed towards the tip interspersed with regular intervals of speeding up and then slowing down again. These previous studies showed that such pulses of movement occur at 2-5 min intervals and it was suggested that they are related to aggregation style pulses of cAMP emitted from the tip of the migrating slug. By sampling the speed of the ventral surface of a moving slug at $15 \mathrm{~s}$ intervals, it was possible to search for the existence of synchronous pulses of movement even if they appeared at intervals as short as $30 \mathrm{~s}$. Our results indicate a much lower frequency (10 $\mathrm{min}$ ) than that previously observed and the shape of the propagating wave detected was also different. In particular, the cells on the ventral surface of the slug moved forward uniformly and at a relatively constant, high speed with intervals of slowing down and then accelerating forward again. Perhaps a more significant correlation for the frequency of wave propagation is the interval between periodic tip raising and lowering as the slug migrates (Breen et al., 1987).

It has long been suspected that the cells within the slug move in a spiral (Clark \& Steck, 1979). Seigert \& Weijer (1992) found spiral movements only at the tip of slugs submerged in mineral oils. Our method detected spirals within the aggregating mound but the movement of the entire ventral layer was parallel to the direction of travel regardless of distance from the tip, confirming the results of Seigert \& Weijer (1992) which were obtained by tracking individual cells at the rear of the slug. From the results reported here, it was seen that the ventral layer of the moving slug in touch with the agar substrate generally travels forward slower than the slug as a whole. These results are in agreement with the results reported by Kakutani \& Takeuchi (1986) and Francis (1962), which also reveal that cells in the ventral layer move forward slightly slower than the slug as a whole. Our results therefore suggest that there is a continual but slow form of cell cycling within the migrating slug; i.e. these slower ventral cells must somehow be recycled, otherwise the slug would continually lengthen and this does not happen (see also Shaffer, 1965; Odell \& Bonner, 1986; Williams et al., 1986).

In the slime trail left behind by the advancing slug there exists, at regular intervals along the trail, clusters of celllike structures that are commonly referred to as 'footprints' (Vardy et al., 1986; Breen \& Williams, 1988; Vardy, 1992). These zones are glycoprotein and cellulose rich and coincide with where the tip of the slug lands on the substratum during migration (Vardy et al., 1986; Breen et al., 1987; Vardy, 1992). Previously (Williams et al., 1986; Breen \& Williams, 1988), it was proposed that cells in the ventral layer of the tip region, when in contact with the substratum would stop and actually attach to the substratum through the liquid ECM at the tip. This attachment of cells to the substratum through the ECM created the 'footprint' zone that is left behind in the slime trail and transiently provided a cellular platform, or 'adhesion-zone', from which the slug could propel itself. Here, we have shown that when the tip of the migrating slug lands on the agar substrate, its ventral cellular surface layer slows, and quickly resumes motion. Hence, 'footprint' zones must be constructed rapidly, probably less than $1 \mathrm{~min}$ after the initial contact period between the ventrum of the tip and the substratum. The cells that secrete this material move on soon afterwards. Our current view is that 'footprints' are regions of ECM strengthening that allow forward propulsion of the slug.

Much of the information relating to cellular movements within tissues of clusters of cells has been obtained from only a few cell types that are amenable to in vitro culture (Trinkaus, 1984; Armstrong, 1985; Fristrom, 1988) or visible because the tissue is transparent (Trinkaus, 1984). It has been argued that in vitro experiments should be interpreted with caution and may not reflect the in vivo situation (Phillips, 1984). The measurements and the observation of moving cells in vivo is difficult as highlighted in the case of the urodele tail and during wound healing in the cat eye (Honda et al., 1982). Because such experiments are rarely automated, the collection of data is labour intensive and usually limited to just a few cells. Experiments on the slug stage of $D$. discoideum suggest that this organism is particularly suitable for the development of the technologies required for the automated analysis of cells within living/moving tissue, as $D$. 
discoideum allows the visualization and the investigation, in vivo, of coordinated cellular movements within a moving eukaryotic tissue. This is particularly true for the tail region of the young WS380B slug and here we have provided evidence of synchrony in behaviour between cells in WS380B slugs, by showing that the vacuoles/nucleus in each cell across the width of the entire tail region of the young WS380B slug can align. This in turn results in a striking transverse banding pattern down the length of the tail of the young WS380B slug. As the lining up of vacuoles across the tail region of young $D$. discoideum slugs has not been reported previously, it provides an explanation for the transverse banding patterns formed by groups of stained cells when they are transplanted into unstained slugs (Bonner, 1957; Farnsworth \& Wolpert, 1971) and suggests that cells within the entire slug line up in a similar manner to that revealed in the tail region of young WS380B slugs.

\section{ACKNOWLEDGEMENTS}

This research was supported by a Program Grant to K.L.W. from the Australian Research Council. E. J. B. was supported by a Commonwealth Postgraduate Research Award. We thank both the reviewers for helpful suggestions. Thanks to P. Soille for useful comments.

\section{REFERENCES}

Armstrong, P. B. (1985). The control of cell motility during embryogenesis. Cancer Metastasis Rev 4, 59-80.

Barnard, S. T. \& Thompson, W. B. (1980). Disparity analysis of images. IEEE Trans Patt Anal Mach Intellig 2, 333-340.

Barnea, D. I. \& Silverman, H. F. (1972). A class of algorithms for fast digital image registration. IEEE Trans Comput 21, 179-186.

Bonner, J. T. (1947). Evidence for the formation of cell aggregates by chemotaxis in the development of the slime mold Dictyostelium discoideum. J Exp Zool 106, 1-26.

Bonner, J. T. (1957). A theory of the control of differentiation in the cellular slime molds. $Q$ Rev Biol 32, 232-246.

Breen, E. J., Eliott, S., Vardy, P. H., White, A. \& Williams, K. L. (1992a). Length regulation in the Dictyostelium discoideum slug is a late event. J Exp Zool 262, 299-306.

Breen, E. J., Joss, G. H. \& Williams, K. L. (1991). Locating objects of interest within biological images: the top hat box filter. $J$ Comput Assisted Microsc 3, 97-101.

Breen, E. J., Joss, G. H. \& Williams, K. L. (1992b). Dynamic arrays for fast, efficient, data manipulation during image analysis: a new software tool for exploratory data analysis. Comput Methods Programs Biomed 37, 85-92.

Breen, E. J., Vardy, P. H. \& Williams, K. L. (1987). Movement of the multicellular slug stage of Dictyostelium discoideum: an analytical approach. Development 101, 313-321.

Breen, E. J. \& Williams, K. L. (1988). Movement of the Dictyostelium slug: models, musings and images. Dev Genet 9, 539-548.

Breen, E. J. \& Williams, K. L. (1990). Contrast matching in a sequence of images by histogram normalization. J Microsc 159, 223-227.

Clark, R. L. \& Steck, T. L. (1979). Morphogenesis in Dictyostelium : an orbital hypothesis. Science 204, 1163-1168.

DemI Rand, K. \& Sussman, M. (1983). The morphogenetic sequence followed by migrating slugs of Dictyostelium discoideum during reentry into the fruiting mode. Differentiation 24, 88-96.

Durston, A. J., Cohen, M. H., Drage, D. J., Potel, M. J., Robertson, A. \& Wonio, D. (1976). Periodic movements of Dictyostelium discoideum sorocarps. Dev Biol 52, 173-180.

Durston, A. J. \& Vork, F. (1979). A cinematographic study of the development of vitally stained Dictyostelium discoideum. J Cell Sci 36, 261-279.

Eliott, S., Vardy, P. H. \& Williams, K. L. (1991). The distribution of myosin II in Dictyostelium discoideum slug cells. J Cell Sci 115, 1267-1276.

Farnsworth, P. A. \& Wolpert, L. (1971). Absence of cell sorting out in the grex of slime mould Dictyostelium discoideum. Nature 231, 329-330.

Firtel, R. A. (1991). Signal transduction pathways controlling multicellular development in Dictyostelium. Trends Genet 7, 381-388.

Francis, D. (1962). Movement of pseudoplasmodia of Dictyostelium discoideum. PhD Diss., Univ. Wisconsin, Madison, USA.

Fristrom, D. (1988). The cellular basis of epithelial morphogenesis: a review. Tissue \& Cell 20, 645-690.

Gelles, J., Schnapp, B. J. \& Sheetz, M. (1988). Tracking kinesindriven movements with nanometre-scale precision. Nature 331, 450-453.

Honda, H., Ogita, Y., Higuchi, S. \& Kani, K. (1982). Cell movements in living mammalian tissue: long term observation of individual cells in wounded corneal endothelia of cats. J Morphol 174, 25-39.

Horn, K. P. \& Schunck, B. G. (1981). Determining optical flow. Art Intellig 17, 185-203.

Inouye, K. \& Takeuchi, I. (1979). Analytical studies on the migrating movement of the pseudoplasmodium of Dictyostelium discoideum. Protoplasma 99, 289-304.

Kakutani, T. \& Takeuchi, I. (1986). Characterization of anterior-like cells in Dictyostelium as analyzed by their movement. Dev Biol 115, 439-445.

Noble, P. B. (1987). Extracellular matrix and cell migration: locomotory characteristics of MOS-11 cells within a threedimensional hydrated collagen lattice. J Cell Sci 87, 241-248.

Odell, G. M. \& Bonner, J. T. (1986). How the Dictyostelium discoideum grex crawls. Philos Trans R Soc Lond B312, 487-525.

Phillips, H. M. (1984). Physical analysis of tissue mechanics in amphibian gastrulation. Am Zool 24, 657-672.

Raper, K. B. (1940). Pseudoplasmodium formation and organization in Dictyostelium discoideum. J Elisha Mitchell $S_{c i} S_{0 c} \mathbf{5 6}$, 241-282.

Robertson, A. D. J. \& Grutsch, J. F. (1981). Aggregation in Dictyostelium discoideum. Cell 24, 603-611.

Rosenfeld, A., Hummel, R. A. \& Zucker, W. W. (1976). Scene labeling by relaxation operations. IEEE Trans Syst Mach Cybernet $\mathbf{6}$, 420-433.

Schaap, P. \& Wang, M. (1984). The possible involvement of oscillatory cAMP signalling in multicellular morphogenesis of the cellular slime molds. Dev Biol 105, 470-478.

Sethi, I. K. \& Jain, R. (1987). Finding trajectories of feature points in a monocular image sequence. IEEE Trans Patt Anal Mach Intellig 9, 56-73.

Shaffer, B. M. (1964). The Acrasina. Adv Morphol 3, 301-322.

Shaffer, B. M. (1965). Cell movement within aggregates of the slime mould Dictyostelium discoideum revealed by surface markers. $J$ Embryol Exp Morpbol 13, 97-117.

Siegert, F. \& Weijer, C. J. (1991). Analysis of optical density wave 
propagation and cell movement in the cellular slime mould Dictyostelium discoideum. Physica D49, 224-232.

Siegert, F. \& Weijer, C. J. (1992). Three-dimensional scroll waves organize Dictyostelium slugs. Proc Natl Acad Sci USA 89, 6433-6437.

Sternfeld, J. \& David, C. N. (1981). Cell sorting during pattern formation in Dictyostelium. Differentiation 20, 10-21.

Sussman, M. (1966). Biochemical and genetic methods in the study of cellular slime mold development. Methods Cell Pbysiol 2, 73-91.

Tasaka, M. \& Takeuchi, I. (1983). Cell patterning during slug migration and early culmination in Dictyostelium discoideum. Differentiation 23, 184-188.

Trinkaus, J. P. (1984). Cells into Organs. Englewood Cliffs, NJ: Princeton-Hall.
Ullman, S. (1981). Analysis of visual motion by biological and computer systems. IEEE Comput Aug, 57-69.

Vardy, P. H., Fisher, L. R., Smith, E. \& Williams, K. L. (1986). Traction proteins in the extracellular matrix of Dictyostelium discoideum slugs. Nature 320, 526-529.

Vardy, P. H. (1992). The extracellular matrix and locomotion in the Dictyostelium discoideum slug. PhD Diss., Macquarie Univ., Sydney, Australia.

Williams, K. L., Vardy, P. H. \& Segel, L. A. (1986). Cell migrations during morphogenesis: some clues from the slug of Dictyostelium discoideum. BioEssays 5, 148-152.

Received 8 June; revised 29 October; accepted 22 November 